\title{
Unified Anisotropic Elastoplastic Model for Sand
}

\author{
Jidong Zhao ${ }^{1}$ and Zhiwei $\mathrm{Gao}^{2}$
}

\begin{abstract}
This paper presents a unified approach to model the influence of fabric anisotropy and its evolution on both the elastic and plastic responses of sand. A physically based fabric tensor is employed to characterize the anisotropic internal structure of sand. It is incorporated into the nonlinear elastic stiffness tensor to describe anisotropic elasticity, and is further included explicitly in the yield function, the dilatancy relation, and the flow rule to characterize the anisotropic plastic sand response. The physical change of fabric with loading is described by a fabric evolution law driven by plastic strain, which influences both the elastic and the plastic sand behavior. The proposed model furnishes a comprehensive consideration of both anisotropic elasticity and anisotropic plasticity, particularly the nonlinear change of elastic stiffness with the evolution of fabric during the plastic deformation of sand. It offers a natural and rational way to capture the noncoaxial behavior in sand caused by anisotropy. It also facilitates easy determination of the initial anisotropy in sand based on simple laboratory tests and avoids the various arbitrary assumptions on its value made by many previous studies. The model predictions on sand behavior compare well with test data. DOI: 10.1061/(ASCE)EM.1943-7889.0000962. () 2015 American Society of Civil Engineers.
\end{abstract}

Author keywords: Sand; Fabric tensor; Anisotropic elasticity and plasticity; Fabric evolution; Critical state; Constitutive model.

\section{Introduction}

Natural soil deposits commonly show a physical feature of cross anisotropy due to natural deposition and/or compaction processes. The physical structure in these soils, known to be soil fabric, exhibits a largely isotropic behavior within the deposition plane and an apparently different behavior along the normal direction to this plane (this normal direction is called the axis of anisotropy) (Miura and Toki 1982; Yoshimine et al. 1998). It has been well documented that the overall behavior of a soil, including its strength, deformation, and failure, is greatly affected by the presence and the change of the anisotropic fabric structure. The bearing capacity of a strip footing composed of crossanisotropic sand, for example, was found to differ by $25 \%$ as much when the load was applied along the axis of anisotropy as compared to the case with load applied in parallel with the deposition plane (Oda et al. 1978; Azami et al. 2010). Because all the other testing conditions were identical, fabric anisotropy caused by sample preparation was considered the major attributable reason for the observed difference (Oda et al. 1978; Azami et al. 2010). The vulnerability of sand to liquefaction was also found closely related to fabric anisotropy in sand. Indeed, both Uthayakumar and Vaid (1998) and Yoshimine et al. (1998) reported that a sand sample under undrained shear may show a dilative and strain hardening behavior in triaxial compression, but may end up with static liquefaction in triaxial extension. The apparent difference between the two cases is the loading direction with respect to the fabric anisotropy in the sample.

The important influence of fabric anisotropy on overall soil behavior has hence drawn increasing attention and has become a focal subject of recent studies on constitutive modeling of sand

\footnotetext{
${ }^{1}$ Associate Professor, Dept. of Civil and Environmental Engineering, Hong Kong Univ. of Science and Technology, Hong Kong, SAR, China (corresponding author). E-mail: jzhao@ust.hk

${ }^{2}$ Lecturer, School of Engineering, Rankine Building, Univ. of Glasgow, Glasgow G12 8LT, U.K.

Note. This manuscript was submitted on May 27, 2014; approved on April 2, 2015; published online on May 28, 2015. Discussion period open until October 28, 2015; separate discussions must be submitted for individual papers. This paper is part of the Journal of Engineering Mechanics, (C) ASCE, ISSN 0733-9399/04015056(12)/\$25.00.
}

(see, e.g., Sekiguchi and Ohta 1977; Pestana and Whittle 1999; Zhang et al. 2007; Li and Dafalias 2002, 2012; Dafalias et al. 2004; Gao et al. 2014, among others). Notably, all these studies placed a predominant focus on the influence of anisotropy on the plastic responses of sand; the effect of anisotropy has been considered either by the rotation of yield surface or by the incorporation of a fabric tensor in the plasticity part of the constitutive relation. Meanwhile, the majority of these studies employed an overly simplified assumption of isotropic elasticity in describing the elastic behavior of sand. In the viewpoint of the authors, however, there are at least three outstanding issues related to the status quo of sand anisotropy modeling.

First, there is compelling experimental evidence indicating that the elastic response of sand is frequently anisotropic due to the physical presence of cross-anisotropic fabric structure formed by vertical compaction/deposition and/or preshearing of sand (or so-called initial anisotropy) (see, e.g., Bellotti et al. 1996; Jiang et al. 1997; Hoque and Tatsuoka 1998; Fioravante 2000; Kuwano and Jardine 2002; Anhdan and Koseki 2005). While the sand behavior is dominantly plastic, proper consideration of elastic stiffness anisotropy under a small strain regime is crucial to the design and evaluation of the operational performance for a wide range of geotechnical structures in which the induced displacement and deformation is small to moderate (Addenbrooke et al. 1997; $\mathrm{Ng}$ et al. 2004; Schädlich and Schweiger 2013). The commonly assumed isotropic elasticity is evidently inadequate to address these issues.

Second, when a sand goes beyond the purely elastic regime and proceeds to the more dominant plastic deformation stage, significant changes in the internal physical fabric structure will occur, which helps the sand to develop optimal resistance to the applied load. This is indeed supported by many recent micromechanical investigations (Zhao and Guo 2013; Guo and Zhao 2013). However, except in only a few recent studies ( $\mathrm{Li}$ and Dafalias 2012; Gao et al. 2014; Wan and Guo 2001; Bauer et al. 2004), the evolving nature of fabric has not been considered in constitutive modeling of sand. The majority of fabric-based sand models have considered a constant fabric during the loading course, which may deviate from both physical and numerical observations. To capture the realistic behavior of fabric anisotropy, a proper fabric evolution law is necessary. 
Indeed pertinent to the above two points, the third issue is concerned with how an evolving fabric affects the elastic response in the plastic deformation stage of a sand. Though the fabric of a sand sample can be initially isotropic, an isotropic nonlinear elasticity assumption is valid to only a certain stage of deformation. When the accumulation of plastic deformation gradually changes the soil fabric, the elastic stiffness will become anisotropic (Ishihara et al. 1975; Kato et al. 2001; Kuwano and Jardine 2002; Gajo et al. 2004; Gajo 2010). Proper characterization of such changes of the elastic stiffness anisotropy during the plastic deformation stage is especially important for modeling the sand behavior in cyclic loading (Lashkari 2010; Li 2002) and strain localization in sand (Bigoni and Loret 1999; Gajo et al. 2004; Gao and Zhao 2013). There have been several attempts to address the fabric effect on the elastic response of sand (Bigoni and Loret 1999; Gajo et al. 2004; Hicher and Chang 2006; Lashkari 2010; Schädlich and Schweiger 2013). For example, Lashkari (2010) proposed a sand model by employing an elastic stiffness tensor expressed in terms of a fabric tensor based on the work by Cowin (1985), while Gajo et al. (2004) introduced an elastic potential dependent on both the fabric tensor and the accumulated plastic strain. However, without proper accounting for the evolving nature of fabric, the effect of fabric anisotropy on the elastic portion of the overall sand response during the plastic deformation regime cannot be fully characterized. A comprehensive and consistent consideration of fabric anisotropy in characterizing both the elastic and plastic behaviors of sand is highly desirable but unavailable.

This study presents a unified elastoplastic sand model accounting for fabric anisotropy and its evolution. The model is based on an anisotropic plasticity model recently proposed by the authors (Gao et al. 2014). Developed within the framework of anisotropic critical state theory ( $\mathrm{Li}$ and Dafalias 2012), the model considered the effect of fabric evolution on plastic sand behavior only. In this study, an anisotropic elastic stiffness tensor expressed in terms of the fabric tensor will be introduced in the model based on the work by Cowin (1985). The same fabric tensor is integrated into the plasticity portion of the model formulation in conjunction with a fabric evolution law, which is driven by the plastic strain. The unified formulation enables one to calibrate the initial degree of anisotropy directly by test data based on the elastic stiffness tensor, and helps to capture the continuous change of elastic stiffness anisotropy with plastic deformation. The model will be verified by comparison of model predictions with the torsional shear test results for Toyoura sand reported in Yoshimine et al. (1998).

\section{Model Formulation}

\section{Anisotropic Elasticity Tensor}

Cowin (1985) proposed the following expression of stiffness tensor $E_{i j k l}$ to describe anisotropic elasticity in a porous medium:

$$
\begin{aligned}
E_{i j k l}= & a_{1} \delta_{i j} \delta_{k l}+a_{2}\left(F_{i j} \delta_{k l}+\delta_{i j} F_{k l}\right) \\
& +a_{3}\left(\delta_{i j} F_{k m} F_{m l}+\delta_{k l} F_{i m} F_{m j}\right)+b_{1} F_{i j} F_{k l} \\
& +b_{2}\left(F_{i j} F_{k m} F_{m l}+F_{i m} F_{m j} F_{k l}\right)+b_{3} F_{i m} F_{m j} F_{k n} F_{n l} \\
& +c_{1}\left(\delta_{k i} \delta_{l j}+\delta_{l i} \delta_{k j}\right)+c_{2}\left(F_{k i} \delta_{l j}+F_{l i} \delta_{k j}+\delta_{k i} F_{l j}+\delta_{l i} F_{k j}\right) \\
& +c_{3}\left(F_{i r} F_{r k} \delta_{l j}+F_{k r} F_{r j} \delta_{l i}+F_{i r} F_{r l} \delta_{k j}+F_{l r} F_{r j} \delta_{i k}\right)
\end{aligned}
$$

where $F_{i j}$ is a second-order fabric tensor representing the anisotropic geometry of internal structure in a soil. The nine coefficients $a_{1}, a_{2}, a_{3}, b_{1}, b_{2}, b_{3}, c_{1}, c_{2}$, and $c_{3}$ are functions of void ratio $e$ and the invariants of $F_{i j}$ and $\delta_{i j}$ (equal to 1 for $i=j$; and 0 for $i \neq j$ ) is the Kronecker delta. As a special case of the more general anisotropic elasticity, Eq. (1) can be used to characterize isotropic, crossanisotropic, and orthotropic elasticity, as has been demonstrated by Cowin (1985). The present study is based on Eq. (1) to consider the anisotropic elasticity in sand. A second-order deviatoric fabric tensor similar to the one used by Li and Dafalias (2012) is employed to characterize the void-based fabric anisotropy in sand ( $\mathrm{Li}$ and $\mathrm{Li}$ 2009). For an initially cross-anisotropic sample with the isotropic plane coinciding with the $x_{2}-x_{3}$ plane and the axis of anisotropy aligning with the $x_{1}$-axis, $F_{i j}$ can be expressed as follows:

$F_{i j}=\left(\begin{array}{ccc}F_{11} & 0 & 0 \\ 0 & F_{22} & 0 \\ 0 & 0 & F_{33}\end{array}\right)=\sqrt{\frac{2}{3}}\left(\begin{array}{ccc}F_{0} & 0 & 0 \\ 0 & -F_{0} / 2 & 0 \\ 0 & 0 & -F_{0} / 2\end{array}\right)$

where $F_{0}(\geq 0)$ is the initial degree of anisotropy. For a general case in which the axes of anisotropy of a sample are not coincident with the reference coordinate system, $F_{i j}$ can be obtained by orthogonal transformation of the expression in Eq. (2). To facilitate the formulation of constitutive equations, $F_{i j}$ is normalized such that its norm $F\left(=\sqrt{F_{i j} F_{i j}}\right)$ is unity and the maximum at the critical state in this study. Despite being general and accurate in describing elastic stiffness anisotropy in sand, Eq. (1) is too cumbersome for practical use and has received various simplifications in constitutive modeling (e.g., Bigoni and Loret 1999; Gajo et al. 2004; Gajo 2010; Lashkari 2010). In this study, Eq. (1) is simplified by neglecting the second and higher order terms of $F_{i j}$, with further assumption of the following relationships for relevant coefficients:

$$
\begin{gathered}
a_{1}=K_{r}-2 G_{r} / 3 \\
a_{2}=\left(K_{r}-2 G_{r} / 3\right) / 2 \\
c_{1}=G_{r} \\
c_{2}=G_{r} / 2
\end{gathered}
$$

where $K_{r}$ and $G_{r}$ denote a reference elastic bulk modulus and a reference elastic shear modulus, respectively, based on the following expressions:

$$
G_{r}=G_{0} \frac{(2.97-e)^{2}}{1+e} \sqrt{p p_{a}}
$$

$$
K_{r}=G_{r} \frac{2(1+\nu)}{3(1-2 \nu)}
$$

where $G_{0}$ is a model parameter and $p_{a}(=101 \mathrm{kPa})$ is the atmospheric pressure; $\nu$ is the Poisson's ratio; and $p$ is the mean normal stress. Consequently, the following simplified elastic stiffness tensor of Eq. (1) is used in this study:

$$
\begin{aligned}
E_{i j k l}= & \left(K_{r}-2 G_{r} / 3\right) \delta_{i j} \delta_{k l}+\left(K_{r}-2 G_{r} / 3\right)\left(F_{i j} \delta_{k l}+\delta_{i j} F_{k l}\right) / 2 \\
& +G_{r}\left(\delta_{k i} \delta_{l j}+\delta_{l i} \delta_{k j}\right)+G_{r}\left(F_{k i} \delta_{l j}+F_{l i} \delta_{k j}+\delta_{k i} F_{l j}+\delta_{l i} F_{k j}\right) / 2
\end{aligned}
$$

It is evident that Eq. (9) can be recovered to the isotropic elastic stiffness tensor when the material fabric is isotropic $\left(F_{i j}=0\right)$. In this case, $K_{r}$ and $G_{r}$ become the commonly referred elastic bulk modulus and shear modulus, respectively. 


\section{Yield Function}

The same fabric-dependent explicit yield function $f$ as assumed in Gao et al. (2014) is employed here:

$$
f=\frac{R}{g(\theta)}-H e^{-k_{h}(A-1)^{2}}=0
$$

where $R=\sqrt{3 / 2 r_{i j} r_{i j}}$ with $r_{i j}=\left(\sigma_{i j}-p \delta_{i j}\right) / p=s_{i j} / p$ being the stress ratio tensor, in which $\sigma_{i j}$ is the stress tensor; $p=$ $\sigma_{i i} / 3$ is the mean normal stress; $s_{i j}$ is the deviator stress tensor; $H$ is a hardening parameter related to the frictional property of sand; $k_{h}$ is a positive model constant; and $g(\theta)$ is an interpolation function based on the Lode angle $\theta$ of $r_{i j}$ as follows:

$$
g(\theta)=\frac{\sqrt{\left(1+c^{2}\right)^{2}+4 c\left(1-c^{2}\right) \sin 3 \theta}-\left(1+c^{2}\right)}{2(1-c) \sin 3 \theta}
$$

where $c=M_{e} / M_{c}$; the ratio between the critical state stress ratio in triaxial extension is $M_{e}$; and that in triaxial compression is $M_{c}$.

In proposing the yield function in Eq. (10), it is assumed that the shear resistance of sand is jointly contributed by the isotropic Coulomb friction and fabric anisotropy. The latter is considered in Eq. (10) by the inclusion of an anisotropic variable $A$ defined by the following joint invariant of the deviatoric fabric tensor $F_{i j}$ and the loading direction tensor $n_{i j}$ (see also Li and Dafalias 2004; Gao et al. 2014):

$$
A=F_{i j} n_{i j}
$$

The deviatoric unit loading direction tensor $n_{i j}$ in Eq. (12) is defined as below following Li and Dafalias (2004) and Gao et al. (2014):

$$
n_{i j}=\frac{N_{i j}-N_{m n} \delta_{m n} \delta_{i j} / 3}{\left\|N_{i j}-N_{m n} \delta_{m n} \delta_{i j} / 3\right\|} \quad \text { with } \quad N_{i j}=\frac{\partial \tilde{f}}{\partial r_{i j}}
$$

where $\tilde{f}=R / g(\theta)$.

\section{Hardening Law and Fabric Evolution}

The model employs the following hardening law for $H$ and evolution law for $F_{i j}$ :

$$
\begin{gathered}
d H=\langle L\rangle r_{h}=\langle L\rangle \frac{G_{r}\left(c_{h}-e\right)}{p}\left[\frac{M_{c} g(\theta) e^{-n \zeta}}{R}-1\right] \\
d F_{i j}=\langle L\rangle \Theta_{i j}=\langle L\rangle k_{f}\left(n_{i j}-F_{i j}\right)
\end{gathered}
$$

where \langle\rangle are the Macauley brackets with $\langle L\rangle=L$ for $L>0$ and $\langle L\rangle=0$ for $L \leq 0$; $L$ is the loading index; $c_{h}, n$, and $k_{f}$ are nonnegative model parameters; and $\zeta$ is the dilatancy state parameter defined as follows (Li and Dafalias 2012):

$$
\zeta=\psi-e_{A}(A-1)
$$

where $e_{A}$ is a model parameter; and $\psi=e-e_{c}$ is the state parameter defined by Been and Jefferies (1985), with $e_{c}$ being the critical state void ratio corresponding to the current mean normal stress $p$. In this model, the critical state line in the $e-p$ plane is given by the three-parameter $\left(e_{\Gamma}, \lambda_{c}\right.$, and $\left.\xi\right)$ formulation proposed by $\mathrm{Li}$ and Wang (1998).

It is noteworthy that the fabric evolution Eq. (15) only affects the plastic sand behavior in the original model developed by the authors (Gao et al. 2014). In the present study, it will also have a crucial influence on changing the elastic stiffness of sand during the plastic loading process, which is self-evident from Eq. (9) in conjunction with Eq. (15). As the plastic shear strain accumulates and the material reaches critical state, the fabric tensor $F_{i j}$ will eventually reach a constant critical value with its orientation being coaxial with the loading direction $n_{i j}$ [Eq. (15)]. This is indeed supported by the distinct-element simulations ( $\mathrm{Li}$ and Li 2009; Zhao and Guo 2013; Guo and Zhao 2013). At the critical state, the degree of elasticity anisotropy will also reach a saturated value according to Eq. (9), which is in agreement with laboratory observations (e.g., Ishihara et al. 1975; Gajo et al. 2004; Lashkari 2010; Gajo 2010).

According to the consistency condition on the yield function Eq. (10) in conjunction with the evolutions of $H$ and $F_{i j}$ expressed in Eqs. (14) and (15), the plastic modulus $K_{p}$ can be obtained as below:

$$
\begin{aligned}
K_{p} & =-\left(\frac{\partial f}{\partial H} r_{h}+\frac{\partial f}{\partial A} \frac{\partial A}{\partial F_{i j}} \Theta_{i j}\right) \\
& =\frac{R}{g(\theta)}\left\{\frac{G_{r}\left(c_{h}-e\right)}{H}\left[\frac{M_{c} g(\theta) e^{-n \zeta}}{R}-1\right]+2 k_{h} k_{f}(1-A)^{2}\right\}
\end{aligned}
$$

\section{Flow Rule and Dilatancy}

An associated noncoaxial flow rule based on the yield function expressed in Eq. (10) is used in this model:

$$
\begin{aligned}
d e_{i j}^{p} & =\langle L\rangle m_{i j}, \quad \text { with } \\
m_{i j} & =\frac{\partial f / \partial r_{i j}-\left(\partial f / \partial r_{m n}\right) \delta_{m n} \delta_{i j} / 3}{\left\|\partial f / \partial r_{i j}-\left(\partial f / \partial r_{m n}\right) \delta_{m n} \delta_{i j} / 3\right\|}
\end{aligned}
$$

where $d e_{i j}^{p}$ is the plastic deviatoric strain increment. Since $\partial f / \partial r_{i j}$ is a function of $r_{i j}$ and $F_{i j}$ and the evolution of $F_{i j}$ is accounted for in the model, the noncoaxial response of sand caused by fabric anisotropy can be naturally described. The readers are referred to Gao et al. (2014) for detailed discussion regarding the noncoaxiality feature offered by the yield function in Eq. (10).

The following fabric-dependent dilatancy relation is used in the model (Li and Dafalias 2000, 2012; Gao et al. 2014):

$$
\begin{aligned}
D & =\frac{d \varepsilon_{v}^{p}}{\left|d \varepsilon_{q}^{p}\right|}=\frac{d \varepsilon_{i i}^{p}}{\sqrt{2 d e_{i j}^{p} d e_{i j}^{p} / 3}} \\
& =\frac{d_{1}}{M_{c} g(\theta)}\left[1+\frac{R}{M_{c} g(\theta)}\right]\left[M_{c} g(\theta) e^{m \zeta}-R\right]
\end{aligned}
$$

where $d_{1}$ and $m$ are two model constants; and $d \varepsilon_{v}^{p}$ and $d \varepsilon_{q}^{p}$ denote the plastic volumetric and shear strain increments, respectively.

\section{Determination of the Initial Degree of Anisotropy $F_{0}$}

It remains difficult to measure the initial anisotropy in soil, especially in the field. There have been attempts to measure the sand particle orientation or void space distribution inside a real sand sample based on various techniques such as image analysis using sliced section or the wave-based measuring of anisotropic shear stiffness to obtain the initial degree of anisotropy (e.g., Oda and Nakayama 1989; Yang et al. 2008). These methods are frequently costly and time-consuming and require specially designed equipment, and most often are fabric-definition specific. They may also cause great disturbance to the tested sample and thus lead to inaccurate measurement of the initial fabric anisotropy. In previous fabric-based studies, $F_{0}$ has commonly been assumed a value 
which appears to be rather arbitrary (e.g., Li and Dafalias 2002 , 2012; Dafalias et al. 2004; Gao et al. 2014). Indeed, this difficulty can be conveniently overcome by the present model with a comprehensive consideration of anisotropic elasticity and plasticity as outlined in the previous sections. Based on the anisotropic elastic stiffness tensor in Eq. (9), one can readily determine $F_{0}$ for use in the present model based on test data in conventional undrained triaxial compression/extension or isotropic compression tests on sand. The calibration procedure is described as follows. For a sand sample with initially cross-anisotropic fabric whose deposition plane coincides with the $x_{2}-x_{3}$ plane such that the fabric tensor can be expressed by Eq. (2), the independent components of the initial anisotropic elasticity tensor in Eq. (9) present the following expressions:

$$
\begin{aligned}
& E_{1111}=\left(K_{r}+4 G_{r} / 3\right)+\sqrt{6} F_{0}\left(K_{r}+4 G_{r} / 3\right) / 3 \\
& E_{2222}=E_{3333}=\left(K_{r}+4 G_{r} / 3\right)-\sqrt{6} F_{0}\left(K_{r}+4 G_{r} / 3\right) / 6 \\
& E_{1122}=E_{1133}=\left(K_{r}-2 G_{r} / 3\right)+\sqrt{6} F_{0}\left(K_{r}-2 G_{r} / 3\right) / 12 \\
& E_{1212}=E_{1313}=\left(1+\sqrt{6} F_{0} / 12\right) G_{r} \\
& E_{2323}=\left(1-\sqrt{6} F_{0} / 6\right) G_{r}
\end{aligned}
$$

As all the five elastic constants on the left-hand side of Eq. (20) can be measured using the small strain tests (see, e.g., Bellotti et al. 1996; Kuwano et al. 2000), one can solve for the three unknowns $K_{r}, G_{r}$, and $F_{0}$ (or equivalently $G_{0}, \nu$, and $F_{0}$ ) based on the lestsquare method. Alternatively, $F_{0}$ can be determined according to the test results at the very beginning of conventional undrained triaxial compression/extension or isotropic consolidation tests on sand where the fabric and stress are initially coaxial. The second method is relatively easier to execute in practice and is recommended in the present study.

When the stress state for the sand sample is initially isotropic before shear (the initial values for both $R$ and $H$ are zero), the model will give purely elastic response at the very beginning of an undrained triaxial compression/extension test (CTC/CTE) as $K_{p}$ is initially infinite [Eq. (17)]. Under triaxial conditions, it is easy to render the major principal stress perpendicular (CTC) or parallel (CTE) to the deposition plane ( $x_{2}-x_{3}$ plane in this study). Consequently, one obtains the following incremental stress-strain relation according to Eqs. (20) and (29):

$$
\begin{aligned}
{\left[\begin{array}{l}
d p \\
d q
\end{array}\right]=} & {\left[\begin{array}{cc}
K_{r} & \left(\sqrt{6} K_{r} / 4+\sqrt{6} G_{r} / 6\right) F_{0} \\
\left(\sqrt{6} K_{r} / 4+\sqrt{6} G_{r} / 6\right) F_{0} & \left(3+\sqrt{6} F_{0} / 2\right) G_{r}
\end{array}\right] } \\
& \cdot\left[\begin{array}{l}
d \varepsilon_{v} \\
d \varepsilon_{q}
\end{array}\right]
\end{aligned}
$$

where $d p\left[=\left(d \sigma_{a}+2 d \sigma_{r}\right) / 3\right]$ is the mean effective stress increment and $d q\left(=d \sigma_{a}-d \sigma_{r}\right)$ is the shear stress increment with $d \sigma_{a}$ and $d \sigma_{r}$ denoting the axial and radial stress increment, respectively; $d \varepsilon_{v}\left(=d \varepsilon_{a}+2 d \varepsilon_{r}\right)$ is the volumetric strain increment and $d \varepsilon_{q}\left[=2\left(d \varepsilon_{a}-d \varepsilon_{r}\right) / 3\right]$ the shear strain increment with $d \varepsilon_{a}$ and $d \varepsilon_{r}$ denoting the axial and radial strain increments, respectively. Since $d \varepsilon_{v}=0$ in undrained loading, the following relation between $d q / d p$ and $F_{0}$ can be obtained according to Eqs. (7), (8), and (21):

$$
\frac{d q}{d p}=\frac{\sqrt{6}}{2} \frac{2 \nu-1}{\nu-2}\left(\frac{6}{F_{0}}+\sqrt{6}\right)
$$

or

Since the Poisson's ratio $\nu(0<\nu<0.5)$ for sand is difficult to obtain and may be dependent on multiple factors including the void ratio, confining pressure, and stress ratio, it is common that a typical value $\nu=0.2$ is assumed for most sand, as it is for the present model (Bellotti et al. 1996; Kuwano and Jardine 2002). Thus, $F_{0}$ can be directly obtained based on the value of $d q / d p$ at the very beginning of an undrained triaxial test and Eq. (23) as follows:

$$
F_{0}=\frac{\sqrt{6}}{(d q / d p)-1}
$$

If the initial effective stress path is perpendicular to the $p$-axis (see Path A in Fig. 1), Eq. (24) gives $F_{0}=0$ as $d q / d p=\infty$, which is consistent with isotropic elasticity. If the sand samples have been prepared via vertical compaction in the laboratory, $d q / d p>1$ is typically observed in triaxial compression and extension tests (e.g., Yoshimine et al. 1998; Finge et al. 2006), and one has $F_{0}>$ 0 according to Eq. (24) (Path B in Fig. 1). Note that $d q>0$ in triaxial compression and $d q<0$ in triaxial extension.

In an isotropic compression test performed in a triaxial cell on a sand sample with the bedding plane being horizontal, the stressstrain relation at the very beginning of loading can also be expressed by Eq. (21). Because $d q=0$ and the relation between $K_{r}$ and $G_{r}$ is expressed by Eq. (8), the following relations can be obtained according to Eq. (21):

$$
\frac{d \varepsilon_{v}}{d \varepsilon_{q}}=\frac{3\left(d \varepsilon_{v} / d \varepsilon_{a}\right)}{3-d \varepsilon_{v} / d \varepsilon_{a}}=-\frac{\sqrt{6}}{2}\left(\frac{6}{F_{0}}+\sqrt{6}\right) \frac{2 \nu-1}{\nu-2}
$$

$$
F_{0}=-\sqrt{6} \frac{(2 \nu-1)\left[\left(d \varepsilon_{v} / d \varepsilon_{a}\right)-3\right]}{3(1-2 \nu)+\left(d \varepsilon_{v} / d \varepsilon_{a}\right)(\nu+1)}
$$

If $\nu=0.2$ is assumed, $F_{0}$ can be obtained according to the initial $d \varepsilon_{v} / d \varepsilon_{a}$ in an isotropic compression test as follows:

$$
F_{0}=\sqrt{6} \frac{\left(d \varepsilon_{v} / d \varepsilon_{a}\right)-3}{2\left(d \varepsilon_{v} / d \varepsilon_{a}\right)+3}
$$

It is evident that $F_{0}=0$ when $d \varepsilon_{v} / d \varepsilon_{a}=3$, which corresponds to the initially isotropic fabric case. Because the sand samples are typically prepared through vertical compaction or pluviation, the elastic stiffness will be bigger in the vertical direction and $d \varepsilon_{v} / d \varepsilon_{a}>3$ (Hoque and Tatsuoka 1998; Lade and Abelev 2005;

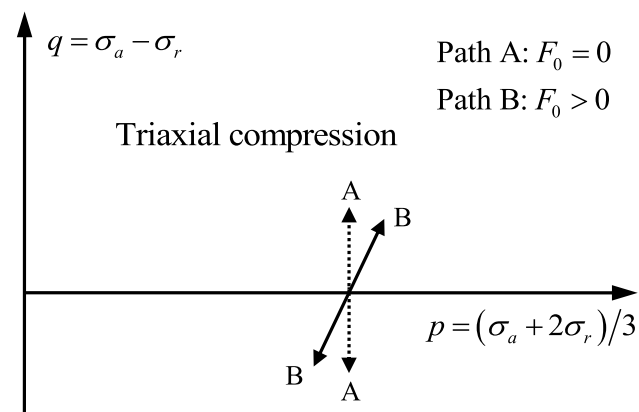

Triaxial extension

Fig. 1. Stress paths at the very beginning of undrained triaxial tests 
Anhdan and Koseki 2005; Finge et al. 2006; Abelev et al. 2007), and, therefore, one has $F_{0}>0$ according to Eq. (27).

\section{Model Verification and Discussion}

In this section, the model will be first verified through a comparison of the model simulations with torsional shear test data on drydeposited Toyoura sand (Yoshimine et al. 1998). The test setup is shown in Fig. 2, in which $\alpha$ denotes the angle between the major principal stress direction and the vertical axis. All the following simulations are based on the calibrated model parameters summarized in Table 1. The parameters are determined using the following procedure:

1. Initial degree of anisotropy $F_{0}: F_{0}$ can be determined based on the small strain tests, isotropic compression tests and triaxial compression tests. Detailed procedure has been discussed in the previous sections. In this paper, the value of $F_{0}(=0.47)$ is evaluated based on the value of $d\left(\sigma_{1}-\sigma_{3}\right) / d p(=6.25)$ at the very beginning of the undrained triaxial compression test ( $\alpha=0^{\circ}$ and $\left.b=0\right)$ using Eq. (24) [Fig. 3(c)]. In the figures, $b\left[=\left(\sigma_{2}-\sigma_{3}\right) /\left(\sigma_{1}-\sigma_{3}\right)\right]$ denotes the intermediate principal stress variable, with $\sigma_{1}, \sigma_{2}$, and $\sigma_{3}$ denoting the major, intermediate, and minor principal stress, respectively.

2. Elastic parameters: The parameter $G_{0}$ can be determined based on the stress-strain relations at the very beginning of triaxial tests. More detailed discussion on this can be found in Taiebat and Dafalias (2008). It is assumed that $\nu=0.2$ in this model.

3. Critical state parameters: The critical state parameters can be obtained directly from the critical state stress ratio in triaxial

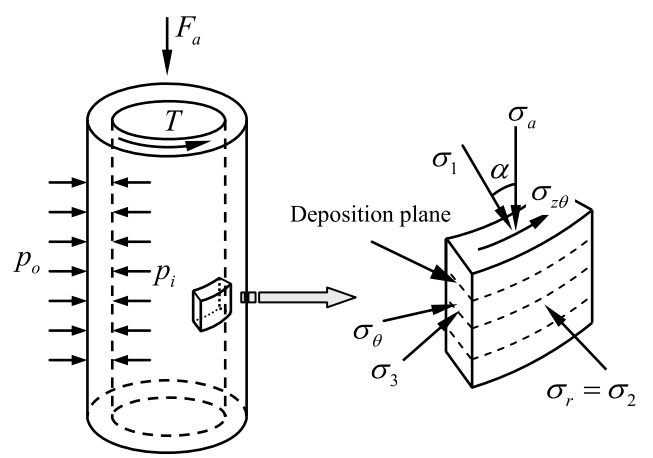

Fig. 2. Torsional shear test setup and stress state for sand element (reprinted from Yoshimine et al. 1998, with permission)

Table 1. Model Parameters for Toyoura Sand

\begin{tabular}{lcc}
\hline Parameter & Symbol & Value \\
\hline Elasticity & $G_{0}$ & 125 \\
Critical state & $\nu$ & 0.2 \\
& $M_{c}$ & 1.25 \\
& $c$ & 0.75 \\
& $e_{\Gamma}$ & 0.934 \\
Plastic modulus & $\lambda_{c}$ & 0.02 \\
& $\xi$ & 0.7 \\
Dilatancy & $c_{h}$ & 1.4 \\
& $n$ & 2.2 \\
Fabric evolution & $d_{1}$ & 0.35 \\
& $m$ & 3.0 \\
& $e_{A}$ & 0.095 \\
\end{tabular}

Note: $F_{0}=0.47$. compression and extension (for $M_{c}$ and $c$ ) and the location of the critical state line in the $e-p$ plane (for $e_{\Gamma}, \lambda_{c}$, and $\xi$ ).

4. Parameters relevant to sand behavior subjected to shear: The parameters $c_{h}, n, d_{1}$, and $m$ can be determined by trial and error to fit the monotonic triaxial compression tests. It is found that $n, d_{1}$, and $m$ are closely related to the particle constitution of sand such as gradation and maximum and minimum void ratio (Gao et al. 2014). Note that $c_{h}$ varies in a small range and only fine tune is needed for different sands to capture the effect of void ratio on plastic hardening of sand in monotonic loading. The parameter $e_{A}$ describes the effect of fabric anisotropy and loading direction on dilatancy and plastic hardening of sand in shear. It can thus be determined by fitting the test results in triaxial extension. It is also found that the variation of $e_{A}$ is small for different sands (Gao et al. 2014).

5. Fabric evolution parameter: Because it is still not possible to measure the fabric evolution in laboratory tests, $k_{f}$ cannot be directly obtained. A feasible way for determining $k_{f}$ is to use the data for noncoaxial sand behavior in simple shear tests (Fig. 8). It is shown by Gao et al. (2014) that $k_{f}$ changes in a small range for different sands.

\section{Model Verification}

Figs. 3 and 4 show the model simulations of the fabric effect on the undrained behavior of sand for $b=0$ and $b=1$, respectively. Clearly, the model predictions agree fairly well with the experimental curves in terms of both the stress-strain relation and the stress path. As $\alpha$ increases, the sand response becomes more contractive while the shear stiffness becomes smaller. Evidently, the present model well captures the inclined stress paths (Path B illustrated in Fig. 1) at the initial loading stage in both figures, which will be further discussed subsequently. Fig. 5 further shows a comparison between the model simulations and the test data for the case of an undrained simple shear test with an initially anisotropic stress state. $K_{0}$ denotes the initial value of $\sigma_{3} / \sigma_{1}$ in the figures. The model clearly captures the experimentally observed sand behavior under undrained simple shear tests. It should be mentioned that there will be numerical problems when the mean effective stress reaches absolute 0 (Fig. 5). In the implementation of the present model, the allowable minimum effective mean stress is set to be $10^{-6}$ rather than exactly 0 to avoid such an issue.

\section{Elasticity Anisotropy Only versus Plasticity Anisotropy Only}

It is interesting to first show how the proposed model facilitates a more accurate description of the behavior observed in sand. For demonstration purpose, the predictions by the elasticity anisotropy only version of the proposed model by setting $k_{f}=$ $e_{A}=0$ (neglecting fabric effect on the plastic response) are compared in Fig. 6 against those by the plasticity anisotropy only version, in which isotropic elasticity is considered (e.g., the model in Gao et al. 2014). Evidently, if isotropic elasticity is assumed (by the plasticity anisotropy only model), the predicted effective stress path at the initial loading stage of an undrained shear test is always perpendicular to the $p$-aixs, exemplified by the four cases in solid curves in Figs. 6(b and d). In contrast, if anisotropic elasticity is employed according to Eq. (9), the predicted slope of the stress path [see the cases in dashed curves in Figs. 6(b and d) at the very beginning of loading], $d\left(\sigma_{1}-\sigma_{3}\right) / d p$, is positive for both testing cases $\left(\alpha=0^{\circ}\right.$ and $\left.45^{\circ}\right)$ at $b=0$ and negative for both cases $\left(\alpha=60^{\circ}\right.$ and $\left.90^{\circ}\right)$ at $b=1$. Note that $d\left(\sigma_{1}-\sigma_{3}\right) / d p=$ $d q / d p$ when $b=0$ and $d\left(\sigma_{1}-\sigma_{3}\right) / d p=-d q / d p$ when $b=1$. 

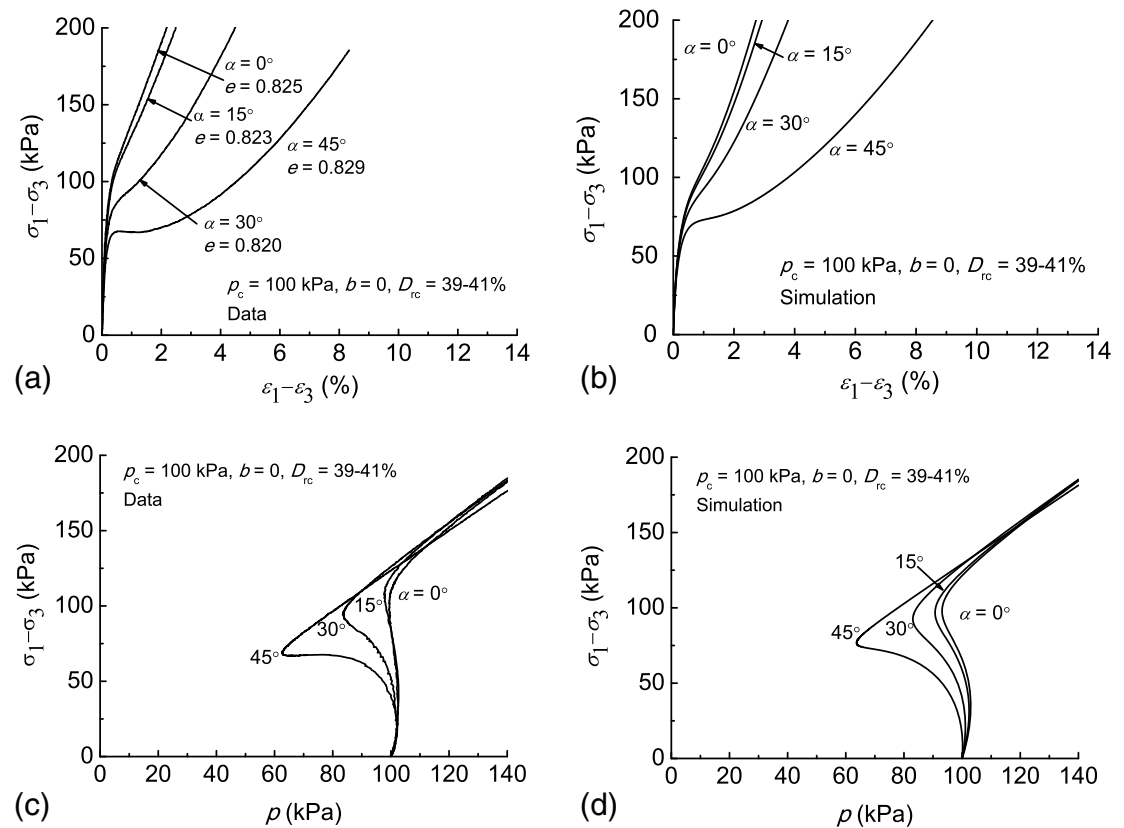

Fig. 3. Test data and model simulations for influence of principal stress direction $\alpha$ on undrained behavior of Toyoura sand at $b=0$ (data from Yoshimine et al. 1998)
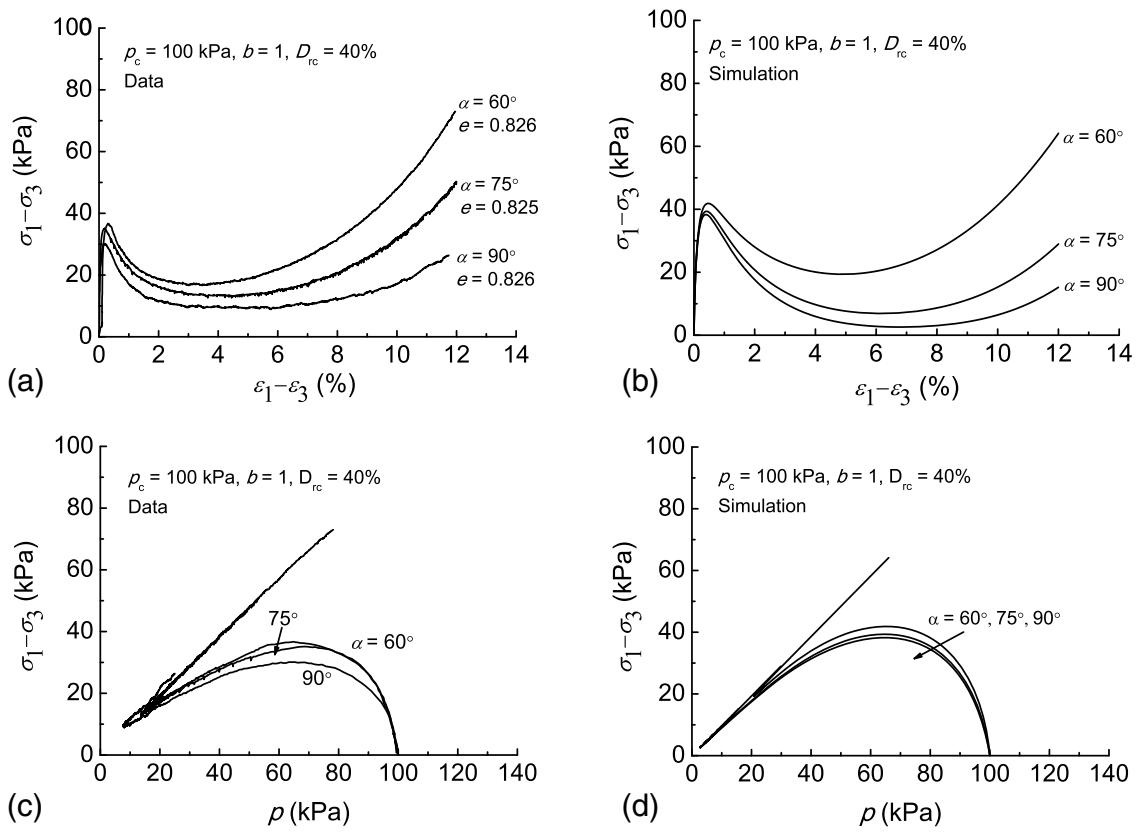

Fig. 4. Test data and model simulations for influence of principal stress direction $\alpha$ on undrained behavior of Toyoura sand at $b=1$ (data from Yoshimine et al. 1998)

In comparison with the test data presented in Figs. 3 and 4, it is evident that the consideration of anisotropic elasticity helps to capture the initial inclination of the effective stress paths considerably better than the isotropic elasticity. The observation is indeed consistent with that reported by Finge et al. (2006).

However, considering the effect of fabric on the elastic sand response alone cannot adequately characterize the overall sand behavior, especially when the shear deformation is large. As seen from Fig. 6, the predictions by the anisotropic elasticity only model apparently lead to pronounced deviations for the predicted stress-strain relation and the effective stress paths from the experimental observations at large shear strains (c.f. test results in Figs. 3 and 4). Note that the effect of anisotropic plasticity is much less significant at relatively small strain level, as can be seen from Fig. 6(e), which shows the same results as Fig. 6(a) at small shear strain level. Proper account of the fabric effect on the plastic sand response is indeed mandatory for realistic modeling of sand behavior at large strain. The present model brings this feature by introducing the anisotropic variable $A$ into the yield function, the hardening law for $H$, as well as the dilatancy relation. Indeed, 

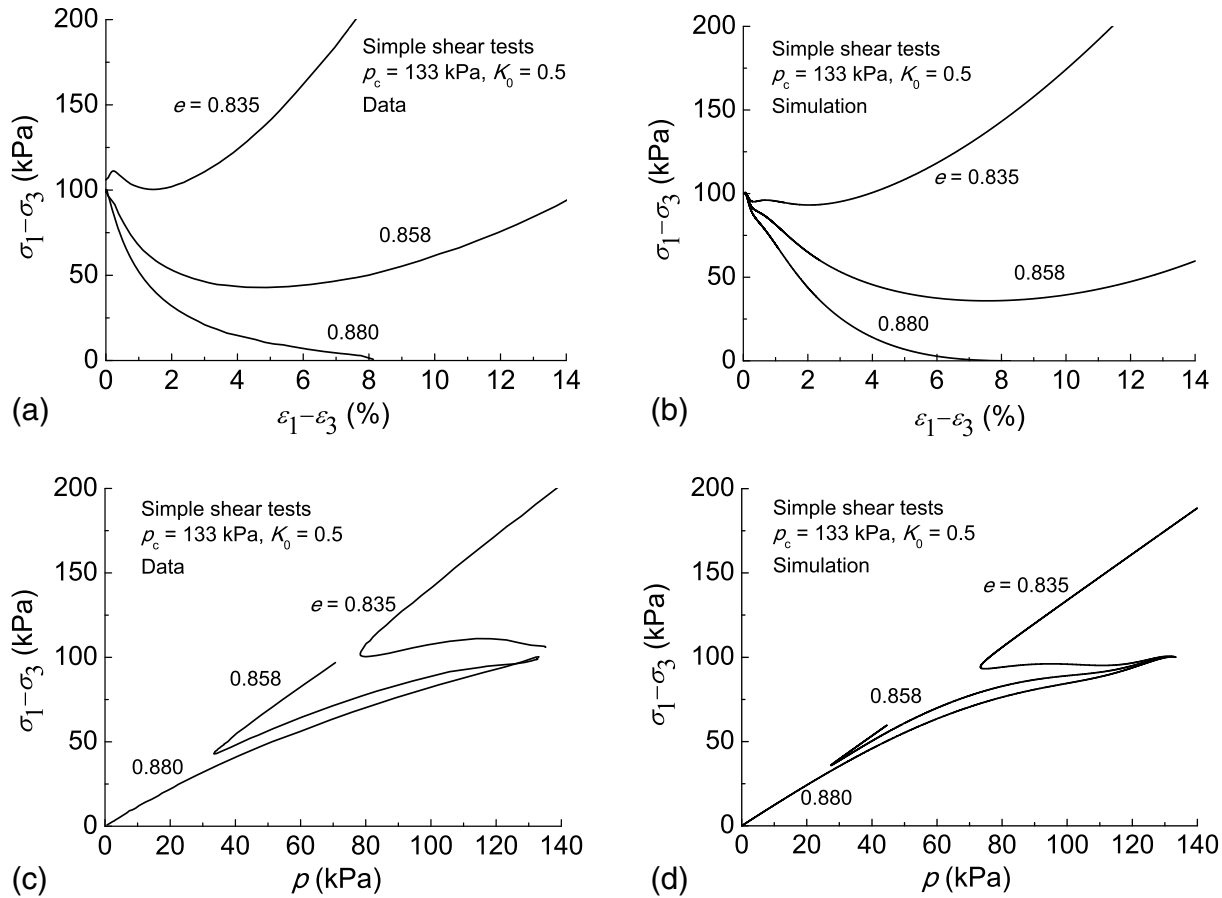

Fig. 5. Comparison between model simulations and undrained simple shear test data on Toyoura sand with initially anisotropic stress state (data from Yoshimine et al. 1998)
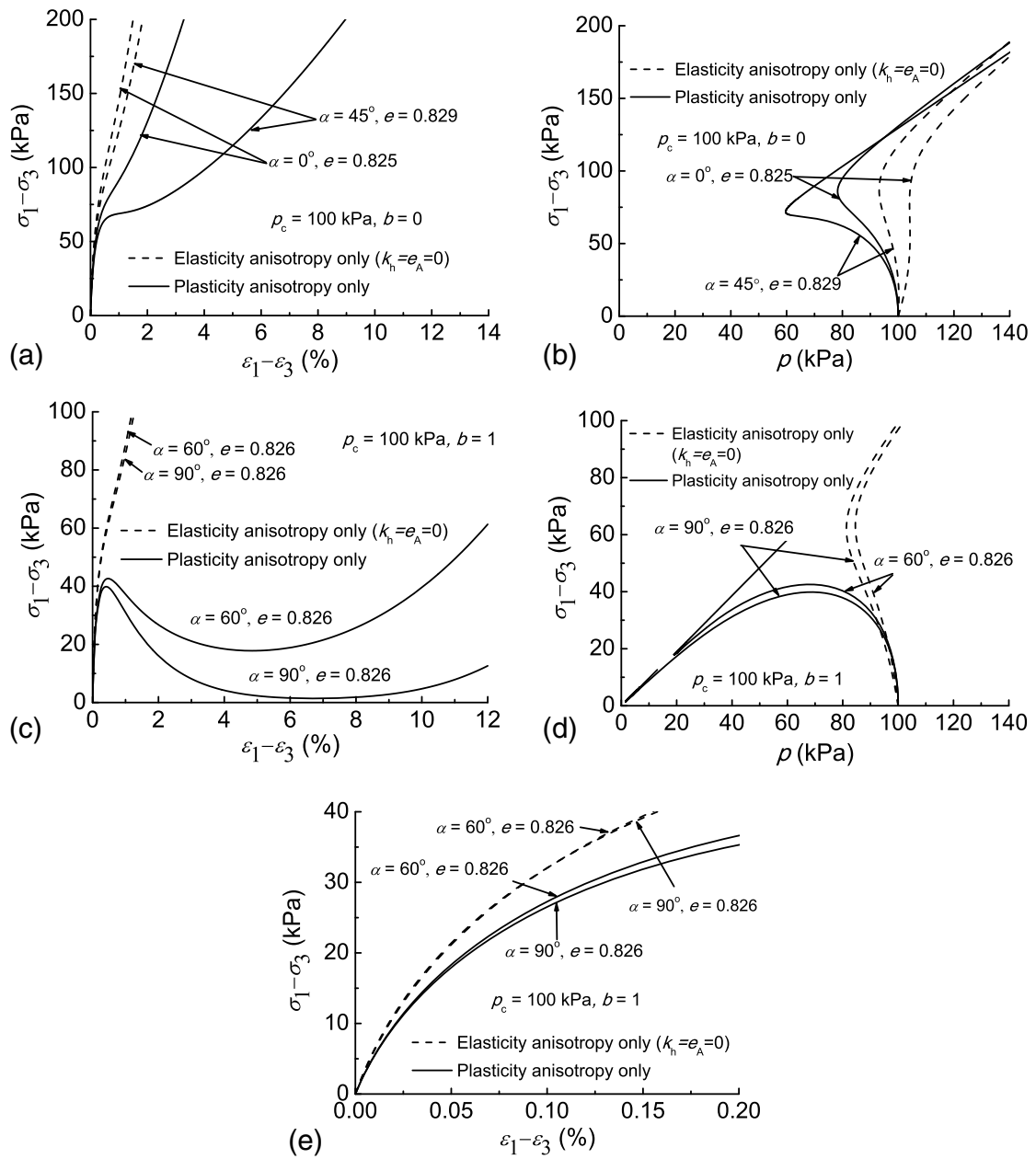

Fig. 6. Effect of anisotropic elasticity on model responses in undrained torsional shear tests: (a) and (b) $b=0$; (c), (d), and (e) $b=1$ 
as is shown in Figs. 3 and 4, the simulations by the present comprehensive anisotropic model exhibit much better agreement with the test data, both at the initial loading stage and at large shear strains.

\section{Unified Description of Fabric Effect on Elastic and Plastic Sand Behavior}

An important feature of the present model, as compared to all past anisotropic sand models including the recent one by the authors (Gao et al. 2014), is its universal consideration of fabric anisotropy and fabric evolution for both elastic and plastic responses of sand behavior. Specifically, the elastic stiffness tensor in the present model changes with plastic strain, due to its dependence via Eq. (9) on the fabric tensor, which evolves with plastic deformation. Because this is readily observed from Fig. 6, it is demonstrated with additional model simulations of undrained triaxial compression with small unloading-reloading cycles at different shear strain levels as shown in Fig. 7. In the simulations the initial sand fabric is assumed to be isotropic $\left(F_{0}=0\right)$. While the stress-strain relations in Fig. 7(a) do not display appreciable differences (Note that the unloading-reloading lines are indeed not vertical in Fig. 7(a). They are seemingly vertical due to the very large strain scale used in plotting the figure to show the entire curve to large strain), the effective stress paths in unloading-reloading after the phase transformation state predicted by the proposed new model [Fig. 7(b)] are distinctly different from those based on isotropic elasticity and anisotropic plasticity (e.g., by Gao et al. 2014). Prior to the phase transformation state, the shear strain is generally small $\left(\varepsilon_{1}-\varepsilon_{3}<3 \%\right)$ and the fabric evolution insignificant [Fig. 7(c)]. Because the considered sample is initially isotropic, the degrees in both the fabric anisotropy and elastic stiffness anisotropy remain small to this stage [Fig. 7(c)]. It is hence not surprising that the predicted unloading-reloading effective stress path is nearly perpendicular to the mean stress axis for both cases [c.f., the plasticity-anisotropyonly case in Figs. 6(b and d)]. After the phase transformation state, the predicted unloading-reloading effective stress paths by the model with isotropic elasticity remain nearly vertical, while the present model based on anisotropic elasticity predicts inclined effective stress paths for the unloading-reloading cycles that are consistent with experimental observations (Ishihara et al. 1975; Verdugo and Ishihara 1996; Gajo 2010; Lashkari 2010). Notably, the inclination angle $\theta$ between the two cases [shown in Fig. 7(b)] increases with the maximum shear strain and ultimately reaches a saturated value at very large shear strain [Fig. 7(b)]. This is also in agreement with the observations reported by Ishihara et al. (1975) and Gajo (2010). The increase of $\theta$ with shear strain is indeed related to the increased degree of elastic stiffness anisotropy, e.g., the ratio of $E_{1111} / E_{3333}$ shown in Fig. $7(\mathrm{c})$, which denotes the ratio of the constraint modulus in the vertical and horizontal directions for the present study. The observed trend in the evolution of $\theta$ indicates that during the monotonic shear, the evolving fabric leads to steadily increased degrees in both the fabric and elastic stiffness anisotropy [Fig. 7(c)]. When the sand sample reaches the critical state at large deformation, the fabric eventually becomes coaxial with the loading direction and reaches a constant magnitude. Consequently, the degree of elastic stiffness anisotropy will cease to evolve further and reaches a saturated value, which is embodied by both $\theta$ and $E_{1111} / E_{3333}$ [Figs. 7(b and c)]. Fig. 7 highlights the important new features associated with the present model that have not been demonstrated by any existing models.

Note that in Fig. 7 the sample has been sheared to a high shear strain level in an attempt to attain critical state (e.g., constant stress, void ratio, and fabric). Indeed, distinct element studies, such as the recent one presented by Fu and Dafalias (2011) and Zhao and Guo (2015), indicate the sand fabric and void ratio inside the shear band can only reach the critical state when the average shear strain in the shear band is well above $200 \%$. The present model has been formulated to offer pure elastic responses during the unloading and reloading process, so it is not capable of capturing the sand behavior in cyclic loading typically with a small elastic domain/nucleus. To this end, the bounding surface concept may be further introduced to generalize this model to describe the sand behavior in cyclic loading, which will be pursued in the future.
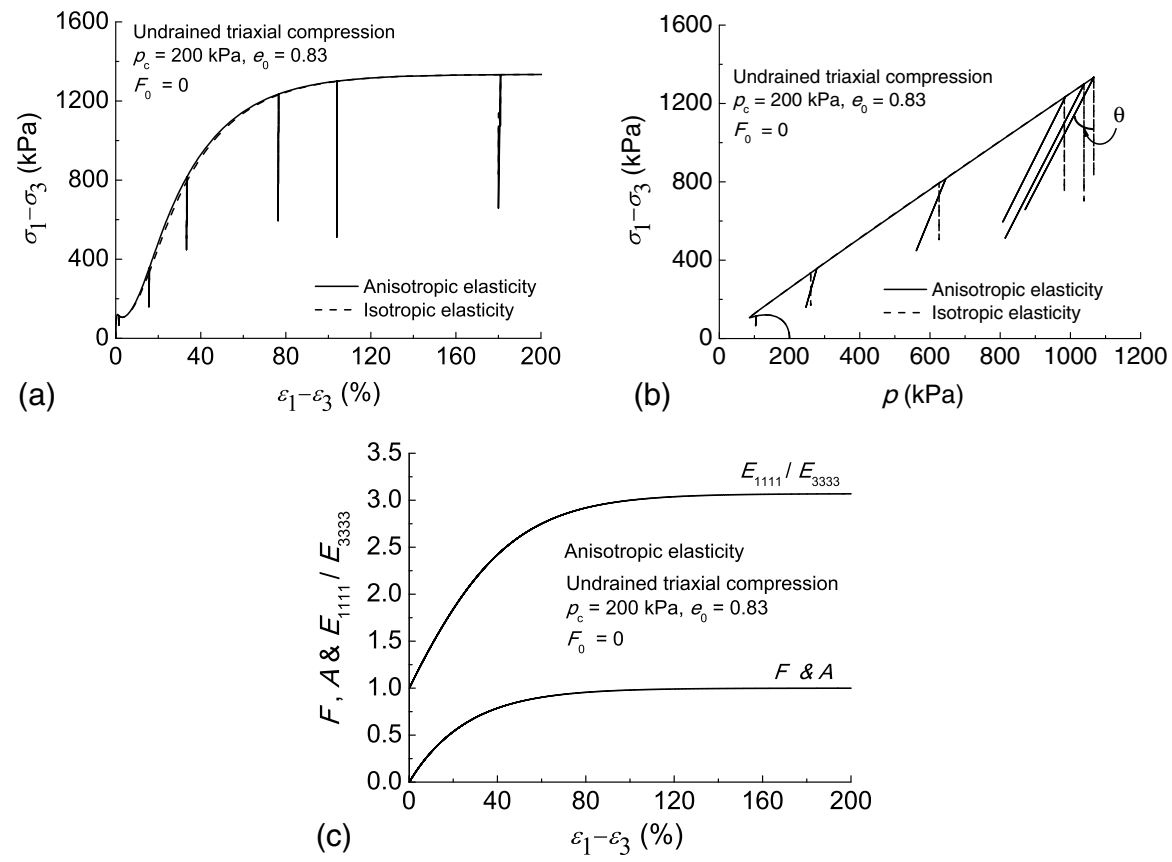

Fig. 7. Effect of elasticity anisotropy evolution on sand response in undrained triaxial compression: (a) shear stress-strain relation; (b) effective stress path; (c) evolution of ratio $E_{1111} / E_{3333}$ with shear strain 

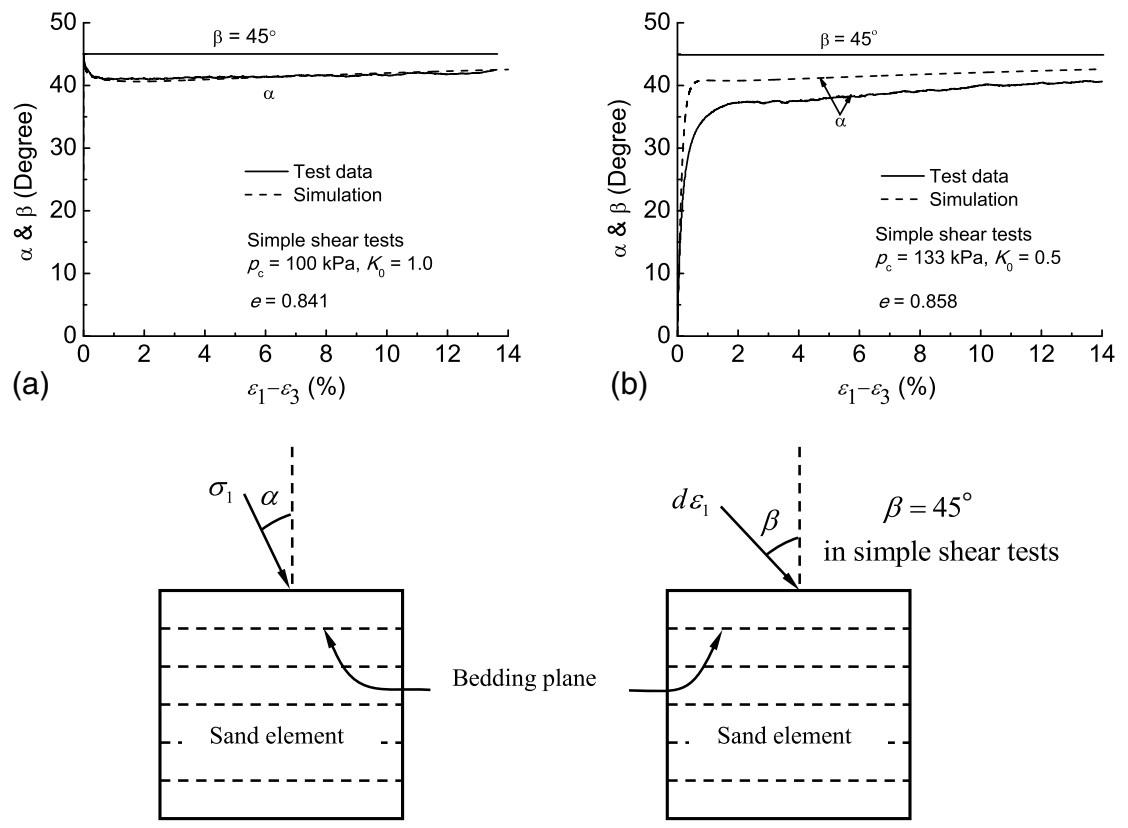

(c)

Fig. 8. (a) and (b) Comparison between tested and simulated noncoaxial sand response in undrained simple shear tests (data from Yoshimine et al. 1998); (c) illustration of relation between $\alpha$ and $\beta$

\section{Prediction of Noncoaxial Sand Behavior in Undrained Simple Shear Tests}

Another feature of the present model is its remarkable capability in predicting noncoaxial behavior in sand. Experimental observations indicate that initial sand response is typically noncoaxial (the major principal axes of the stress and strain increment do not coincide) if the sand fabric and loading direction are not coaxial at the beginning of loading, and the degree of noncoaxiality decreases gradually with shear strain and vanishes at large deformation (Roscoe 1970; Yoshimine et al. 1998; Thornton and Zhang 2006). There have been various attempts toward modeling such unique sand behavior, e.g., by assuming that the plastic strain increment is dependent on both the current stress state and the stress increment direction (e.g., Gutierrez et al. 1993; Yu and Yuan 2006). Most often, the physical significance behind these approaches in treating noncoaxiality is unclear. The present model can describe such sand response in a rather natural and physically plausible manner. In the plasticity part, as entailed in Gao et al. (2014) and also discussed in Zhao and Guo (2013), the employment of an associated noncoaxial flow rule [Eq. (18)] based on a fabric-dependent yield function [Eq. (10)] leads to a natural fraction of contribution of noncoaxial fabric to the total plastic strain, which facilitates the modeling of noncoaxiality.

As an illustrative example, Fig. 8 shows the model simulations on the noncoaxial response in undrained simple shear tests on sand with initially isotropic [Figs. 8(a and c)] and anisotropic [Figs. 8(b and c)] stress states, where $\beta$ ( $\equiv 45^{\circ}$ in simple shear tests) denotes the orientation of the major principal strain increment direction relative to the vertical axis and $\alpha$ is the angle between the major principal stress direction and the vertical axis [Fig. 8(c)]. In the case with an initially isotropic stress state [Fig. 8(a)], the model well captures the evolution of $\alpha$. When the initial stress state is anisotropic, the model prediction deviates moderately from the test data but can still reasonably describe the continuous increase trend of $\alpha$ toward $\beta$. For both cases, $\alpha$ will finally reach an identical value of $\beta$ at the critical state when the fabric and loading direction are totally coaxial and the noncoaxial strain increment vanishes.

\section{Prediction of Effect of Sample Preparation Method on Sand Behavior}

Numerous experimental investigations indicate sand response is strongly affected by the sample preparation method (e.g., Miura and Toki 1982; Vaid et al. 1999; Ishihara 1993; Papadimitriou et al. 2005; Yang et al. 2008; Lee et al. 1999; Sze and Yang 2014). Because the sand samples are typically prepared through vertical compaction or pluviation, the sand fabrics created by the various preparation methods are commonly cross-anisotropic but with varied degrees of initial anisotropy. The present model is able to capture the influence of sample preparation method on the sand response, which is showcased in Fig. 9 with examples in drained and undrained triaxial tests. Note that $\sigma_{a}-\sigma_{r} \geq 0$ in triaxial compression and $\sigma_{a}-\sigma_{r} \leq 0$ in triaxial extension. An initially isotropic fabric case $\left(F_{0}=0\right)$ is compared to an anisotropic fabric case $\left(F_{0}=0.47\right)$. Compared to the isotropic sample, the prediction on the initially anisotropic sample shows a higher shear resistance and a more dilative response in triaxial compression and displays a lower shear resistance and more contractive response in triaxial extension. The predicted responses are consistent with experimental observations, e.g., by Sze and Yang (2014), in which the drydeposited samples behave like the initially anisotropic case and the moist-tamped ones behave like the initially isotropic case. Indeed, it is found that the dry-deposited samples have a higher initial degree of anisotropy (Yang et al. 2008). The phenomenon can be easily explained with the present model too. The initial anisotropic parameter $A$ for the initially anisotropic sand sample is respectively bigger and smaller in triaxial compression and extension than the isotropic case. Noting that both the relative orientation between fabric and loading direction and the initial degree of fabric anisotropy contribute to $A$, it is the different initial $A$ that causes the different responses in Fig. 9. Also notably, the differences between initially anisotropic and isotropic fabric cases decrease with loading progress and totally vanish as the fabric in both cases evolve toward the same critical state value [Fig. $9(\mathrm{~g})]$. To further demonstrate this, the evolution of $K_{p}$ and $D$ for cases with $F_{0}=0$ and 0.47 in drained triaxial compression is shown in Figs. 9 (e and f). 


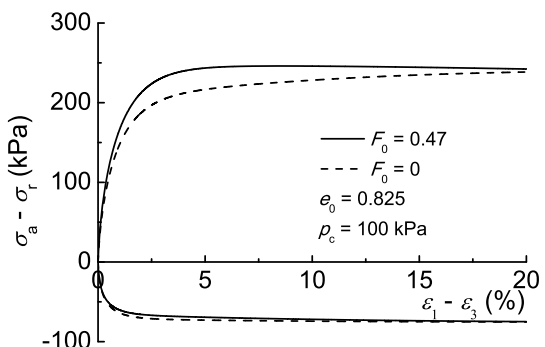

(a)

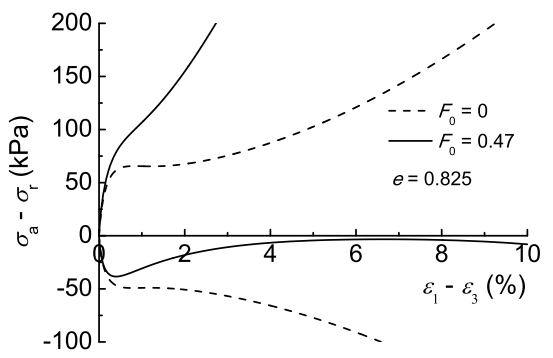

(c)

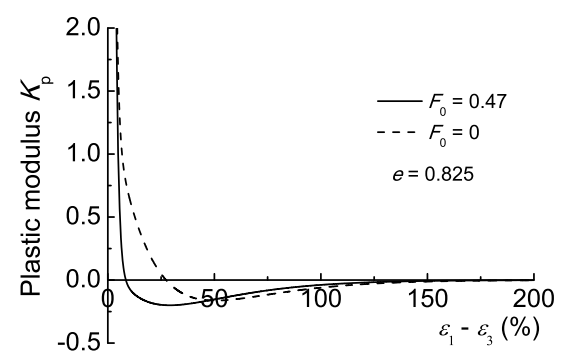

(e)

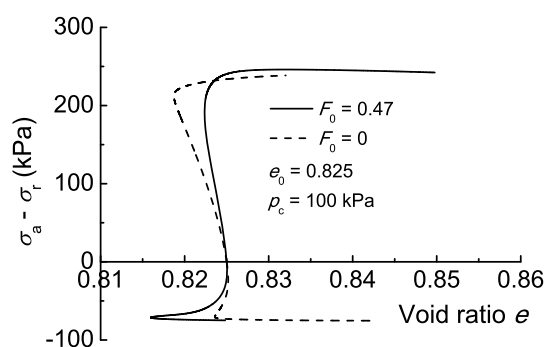

(b)

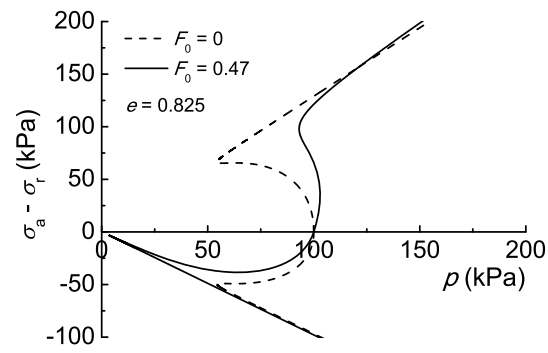

(d)

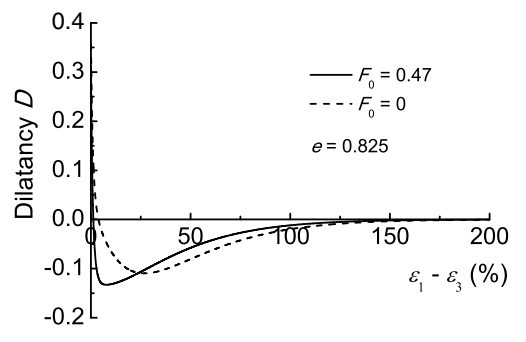

(f)

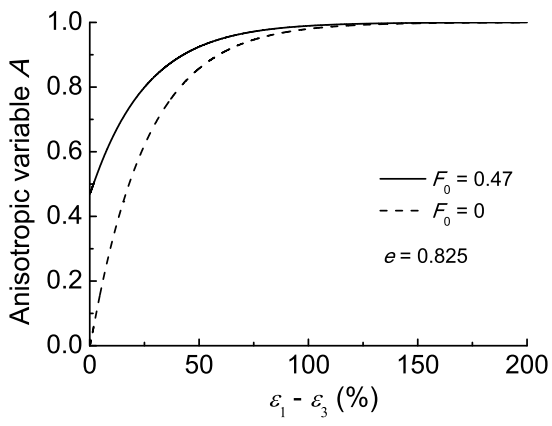

(g)

Fig. 9. Model simulation for effect of sample preparation method on sand behavior in (a) and (b) drained triaxial tests; (c) and (d) undrained triaxial test; (e)-(g) evolution of $K_{p}, D$, and $A$ for cases with $F_{0}=1$ and 0.47 in drained triaxial compression

As $A$ approaches the critical state value 1 for both cases, the differences between $K_{p}$ and $D$ for the two become smaller [Figs. 9(e and f)]. At the critical state (e.g., $\varepsilon_{1}-\varepsilon_{3}=200 \%$ ), the values of $K_{p}$ and $D$ for both cases become $0(A=1$ for both cases). For more discussion of the influence of $A$ on sand response, please refer to Gao et al. (2014).

\section{Conclusions}

A unified approach has been proposed to model the fabric effect on both elastic and plastic behavior of sand. The proposed model has the following main features:

1. The employment of a fabric-dependent anisotropic elastic stiffness tensor helps to realistically reproduce both the initial anisotropic sand response and the steady change of elastic behavior with plastic deformation through the evolution of fabric with plastic shear strain. At the critical state, the fabric tensor is coaxial with the loading direction and reaches unity in magnitude, which leads to a saturated degree of elastic stiffness anisotropy.

2. The effect of fabric and its evolution on plastic sand behavior is considered through explicit inclusion of the fabric tensor in the yield function, the dilatancy relation, and the flow rule. In particular, the flow rule can naturally account for the noncoaxial behavior of sand under monotonic loading.

3. The unified consideration of anisotropic elasticity and anisotropic plasticity not only offers integrated and seamless modeling of sand behavior from small to large strain, but also facilitates the calibration of initial anisotropy based on simply 
designed laboratory tests. This helps to avoid arbitrariness in assuming the initial fabric commonly existing in most previous studies.

The model simulations have been compared with undrained torsional shear test results on Toyoura sand (Yoshimine et al. 1998), with good agreements observed. The model can capture the inclined effective stress path at the very beginning of conventional undrained triaxial tests (when the fabric and stress are initially coaxial) typically observed in laboratory tests (Yoshimine et al. 1998) as well as the evolution of anisotropic elasticity with loading history. Specifically, the study illustrates that the degree of anisotropy in the elastic stiffness increases with the plastic shear strain and reaches a saturated value at very large strain, which is mainly due to the fabric evolution with plastic deformation. At the critical state, the fabric is codirectional with the loading direction and reaches a constant magnitude, resulting in a constant degree of anisotropy in elastic stiffness, which depends on the fabric tensor. In addition, the consideration of fabric and fabric evolution in the plasticity part of a model is shown to be essential for realistic modeling of the anisotropic sand response at large strain. With further illustrative examples, the model has been shown to capture the noncoaxial behavior in sand and the effect of sample preparation method on sand behavior with reasonable agreement with experimental observations. While the present model has been developed for the monotonic loading case, it remains exploratory to improve it to furnish modeling of cyclic behavior of sand.

\section{Appendix. Constitutive Equations}

The condition of consistency for the yield function can be expressed as

$$
d f=\frac{\partial f}{\partial \sigma_{i j}} d \sigma_{i j}+\frac{\partial f}{\partial H} d H+\frac{\partial f}{\partial F_{i j}} d F_{i j}=\frac{\partial f}{\partial \sigma_{i j}} d \sigma_{i j}-\langle L\rangle K_{p}=0
$$

where $K_{p}$ is shown in Eq. (17) and

$$
d \sigma_{i j}=E_{i j k l} d \varepsilon_{k l}^{e}
$$

where $d \varepsilon_{i j}^{e}$ is the elastic strain increment and $E_{i j k l}$ is expressed by Eq. (9). According to Eqs. (18) and (9), the plastic strain increment $d \varepsilon_{i j}^{p}$ can be calculated as below:

$$
\begin{aligned}
d \varepsilon_{i j}^{p} & =d e_{i j}^{p}+d \varepsilon_{v}^{p} \delta_{i j} / 3=\langle L\rangle m_{i j}+\langle L\rangle D \sqrt{2 / 3 m_{k l} m_{k l}} \delta_{i j} / 3 \\
& =\langle L\rangle \underbrace{\left(m_{i j}+\sqrt{6} / 9 D \delta_{i j}\right)}_{x_{i j}}
\end{aligned}
$$

Based on the additive decomposition of the total strain increment

$$
d \varepsilon_{i j}=d \varepsilon_{i j}^{e}+d \varepsilon_{i j}^{p}
$$

and Eqs. (28)-(31), one can get

$$
d f=\frac{\partial f}{\partial \sigma_{i j}} E_{i j k l}\left(d \varepsilon_{k l}-\langle L\rangle x_{k l}\right)-\langle L\rangle K_{p}=0
$$

and thus

$$
L=\underbrace{\frac{\left(\partial f / \partial \sigma_{i j}\right) E_{i j k l}}{K_{p}+\left(\partial f / \partial \sigma_{i j}\right) E_{i j k l} x_{k l}}}_{\Pi_{k l}} d \varepsilon_{k l}
$$

Combining Eqs. (29), (31), and (33), the constitutive equation can be obtained as below:

$$
d \sigma_{i j}=\Lambda_{i j k l} d \varepsilon_{k l}
$$

where

$$
\Lambda_{i j k l}=E_{i j k l}-h(d L)\left(E_{i j m n} x_{m n}\right) \Pi_{k l}
$$

where $h(d L)$ is the Heaviside step function, with $h(d L>0)=1$ and $h(d L \leq 0)=0$.

The expressions for $\partial f / \partial H, \partial f / \partial F_{i j}, n_{i j}$ (or $N_{i j}$ ), and $m_{i j}$ (or $\left.\partial f / \partial r_{i j}\right)$ are shown in Gao et al. (2014) and

$$
\frac{\partial f}{\partial \sigma_{i j}}=\frac{\partial f}{\partial r_{i j}}\left(\frac{\delta_{k i} \delta_{l j}}{p}-\frac{\sigma_{k l}}{3 p^{2}} \delta_{i j}\right)
$$

\section{Acknowledgments}

The authors wish to thank Prof Xiang-Song Li for his comments on the study, as well as the constructive comments offered by the two anonymous reviewers. This work was supported by the Research Grants Council of Hong Kong (under grant No. 623211) and HKUST Postdoctoral Fellowship Matching Fund.

\section{References}

Abelev, A. V., Gutta, S. K., Lade, P. V., and Yamamuro, J. A. (2007). "Modeling cross-anisotropy in granular materials." J. Eng. Mech., 10.1061/ (ASCE)0733-9399(2007)133:8(919), 919-932.

Addenbrooke, T. I., Potts, D. M., and Puzrin, A. M. (1997). "The influence of pre-failure soil stiffness on the numerical analysis of tunnel construction." Géotechnique, 47(3), 693-712.

Anhdan, L., and Koseki, J. (2005). "Small strain behaviour of dense granular soils by true triaxial tests." Soils Found., 45(3), 21-38.

Azami, A., Pietruszczak, S., and Guo, P. (2010). "Bearing capacity of shallow foundations in transversely isotropic granular media." Int. J. Numer. Anal. Methods Geomech., 34(8), 771-793.

Bauer, E., Huang, W. X., and Wu, W. (2004). "Investigations of shear banding in an anisotropic hypoplastic material." Int. J. Solids Struct., 41(21), 5903-5919.

Been, K., and Jefferies, M. G. (1985). "A state parameter for sands." Géotechnique, 35(2), 99-112.

Bellotti, R., Jamiolkowski, M., Lo Presti, D. C. F., and O'Neill, D. A. (1996). "Anisotropy of small strain stiffness in Ticino sand." Géotechnique, 46(1), 115-131.

Bigoni, D., and Loret, B. (1999). "Effects of elastic anisotropy on strain localization and flutter instability in plastic solids." J. Mech. Phys. Solids, 47(7), 1409-1436.

Cowin, S. C. (1985). "The relationship between the elasticity tensor and the fabric tensor." Mech. Mater., 4(2), 137-147.

Dafalias, Y. F., Papadimitriou, A. G., and Li, X. S. (2004). "Sand plasticity model accounting for inherent fabric anisotropy." J. Eng. Mech., 10.1061/(ASCE)0733-9399(2004)130:11(1319), 1319-1333.

Finge, Z., Doanh, T., and Dubujet, P. (2006). "Undrained anisotropy of Hostun RF loose sand: New experimental investigations." Can. Geotech. J., 43(11), 1195-1212.

Fioravante, V. (2000). "Anisotropy of small stain stiffness of Ticino and Kenya sands from seismic propagation measured in triaxial testing." Soils Found., 40(4), 129-142.

Fu, P. C., and Dafalias, Y. F. (2011). "Fabric evolution within shear bands of granular materials and its relation to critical state theory." Int. J. Numer. Anal. Methods Geomech., 35(18), 1918-1948. 
Gajo, A. (2010). "Hyperelastic modelling of small-strain stiffness anisotropy of cyclically loaded sand." Int. J. Numer. Anal. Methods Geomech., 34(2), 111-134.

Gajo, A., Bigoni, D., and Muir Wood, D. (2004). "Multiple shear band development and related instabilities in granular materials." J. Mech. Phys. Solids, 52(12), 2683-2724.

Gao, Z. W., and Zhao, J. D. (2013). "Strain localization and fabric evolution in sand." Int. J. Solids Struct., 50(22-23), 3634-3648.

Gao, Z. W., Zhao, J. D., Li, X. S., and Dafalias, Y. F. (2014). "A critical state sand plasticity model accounting for fabric evolution." Int. J. Numer. Anal. Methods Geomech., 38(4), 370-390.

Guo, N., and Zhao, J. D. (2013). "The signature of shear-induced anisotropy in granular media." Comput. Geotech., 47, 1-15.

Gutierrez, M., Ishihara, K., and Towhata, I. (1993). "Model for the deformation of sand during rotation of principal stress directions." Soils Found., 33(3), 105-117.

Hicher, P. Y., and Chang, C. S. (2006). "Anisotropic nonlinear elastic model for particulate materials." J. Geotech. Geoenviron. Eng., 10.1061/ (ASCE)1090-0241(2006)132:8(1052), 1052-1061.

Hoque, E., and Tatsuoka, F. (1998). "Anisotropy in elastic deformation of granular materials." Soils Found., 38(1), 163-179.

Ishihara, K. (1993). "Liquefaction and flow failure during earthquakes." Géotechnique, 43(3), 351-451.

Ishihara, K., Tatsuoka, F., and Yasuda, S. (1975). "Undrained deformation and liquefaction of sand under cyclic stresses." Soils Found., 15(1), 29-44.

Jiang, G. L., Tatsuoka, F., Fora, A., and Koseki, J. (1997). "Inherent and stress-state-induced anisotropy in very small strain stiffness of a sandy gravel." Géotechnique, 47(3), 509-521.

Kato, S., Ishihara, K., and Towhata, I. (2001). "Undrained shear characteristics of sand under anisotropic consolidation." Soils Found., 41(1), $1-11$.

Kuwano, R., Connolly, T. M., and Jardine, R. J. (2000). "Anisotropic stiffness measurements in a stress-path triaxial cell." Geotech. Test J., 23(2), 141-157.

Kuwano, R., and Jardine, R. J. (2002). "On the applicability of crossanisotropic elasticity to granular materials at very small strains." Géotechnique, 52(10), 727-749.

Lade, P. V., and Abelev, A. (2005). "Characterization of cross-anisotropic soil deposits from isotropic compression tests." Soils Found., 45(5), 89-102.

Lashkari, A. (2010). "A SANISAND model with anisotropic elasticity." Soil Dyn. Earthquake Eng., 30(12), 1462-1477.

Lee, K. M., Shen, C. K., Leung, D. H. K., and Mitchell, J. K. (1999). "Effects of placement method on geotechnical behavior of hydraulic fill sands." J. Geotech. Geoenviron. Eng., 10.1061/(ASCE)1090-0241 (1999)125:10(832), 832-846.

Li, X. S. (2002). "A sand model with state dependent dilatancy." Géotechnique, 52(3), 173-186.

Li, X. S., and Dafalias, Y. F. (2000). "Dilatancy for cohesionless soils." Géotechnique, 50(4), 449-460.

Li, X. S., and Dafalias, Y. F. (2002). "Constitutive modeling of inherently anisotropic sand behavior." J. Geotech. Geoenvir. Eng., 128(10), 868880.

Li, X. S., and Dafalias, Y. F. (2004). "A constitutive framework for anisotropic sand including non-proportional loading." Géotechnique, 54(1), $41-55$.

Li, X. S., and Dafalias, Y. F. (2012). "Anisotropic critical state theory: The role of fabric." J. Eng. Mech., 10.1061/(ASCE)EM.1943-7889 $.0000324,263-275$.

Li, X. S., and Li, X. (2009). "Micro-macro quantification of the internal structure of granular materials." J. Eng. Mech., 10.1061/(ASCE) 0733-9399(2009)135:7(641), 641-656.
Li, X. S., and Wang, Y. (1998). "Linear representation of steady-state line for sand.” J. Geotech. Geoenviron. Eng., 10.1061/(ASCE)1090-0241 (1998)124:12(1215), 1215-1217.

Miura, S., and Toki, S. (1982). "A sample preparation method and its effect on static and cyclic deformation-strength properties of sand." Soils Found., 22(1), 61-77.

Ng, C. W. W., Leung, E. H. Y., and Lau, C. K. (2004). "Inherent anisotropic stiffness of weathered geomaterial and its influence on ground deformations around deep excavations." Can. Geotech. J., 41(1), 12-24.

Oda, M., Koishikawa, I., and Higuchi, T. (1978). "Experimental study of anisotropic shear strength of sand by plane strain test." Soils Found., $18(1), 25-38$.

Oda, M. and Nakayama, H. (1989). "Yield function for soil with anisotropic fabric." J. Eng. Mech., 10.1061/(ASCE)0733-9399(1989)115: 1(89), 89-104.

Papadimitriou, A. G., Dafalias, Y. F., and Yoshimine, M. (2005). "Plasticity modeling of the effect of sample preparation method on sand response." Soils Found., 45(2), 109-123.

Pestana, J. M., and Whittle, A. J. (1999). "Formulation of a unified constitutive model for clays and sands." Int. J. Numer. Anal. Methods Geomech., 23(12), 1215-1243.

Roscoe, K. H. (1970). "The influence of strains in soil mechanics." Géotechnique, 20(2), 129-170.

Schädlich, B., and Schweiger, H. F. (2013). "The influence of anisotropic small strain stiffness on the deformation behavior of geotechnical structures." Int. J. Geomech., 10.1061/(ASCE)GM.1943-5622.0000286, 861-868.

Sekiguchi, H., and Ohta, K. (1977). "Induced anisotropy and time dependency in clays." Constitutive Equations of Soils, Proc., 9th Int. Conf. on Soil Mechanics and Foundation Engineering, Japanese Society of Soil Mechanics and Foundation Engineering, Tokyo, 229-238.

Sze, H., and Yang, J. (2014). "Failure modes of sand in undrained cyclic loading: Impact of sample preparation." J. Geotech. Geoenviron. Eng., 10.1061/(ASCE)GT.1943-5606.0000971, 152-169.

Taiebat, M., and Dafalias, Y. F. (2008). "SANISAND: Simple anisotropic sand plasticity model." Int. J. Numer. Anal. Methods Geomech., 32(8), 915-948.

Thornton, C., and Zhang, L. (2006). "A numerical examination of shear banding and simple shear non-coaxial flow rules." Philos. Mag., 86(21), 3425-3452.

Uthayakumar, M., and Vaid, Y. P. (1998). "Static liquefaction of sands under multiaxial loading." Can. Geotech. J., 35(2), 273-283.

Vaid, Y. P., Sivathayalan, S., and Stedman, D. (1999). "Influence of specimen-reconstituting method on the undrained response of sand." Geotech. Test. J., 22(3), 187-195.

Verdugo, R., and Ishihara, K. (1996). "Steady state of sandy soils." Soils Found., 36(2), 81-91.

Wan, R. G., and Guo, P. J. (2001). "Effect of microstructure on undrained behaviour of sands." Can. Geotech. J., 38(1), 16-28.

Yang, Z. X., Li, X. S., and Yang, J. (2008). "Quantifying and modelling fabric anisotropy of granular soils." Géotechnique, 58(4), 237-248.

Yoshimine, M., Ishihara, K., and Vargas, W. (1998). "Effects of principal stress direction and intermediate principal stress on undrained shear behaviour of sand." Soils Found., 38(3), 179-188.

Yu, H. S., and Yuan, X. (2006). "On a class of non-coaxial plasticity models for granular soils." Proc. R. Soc. A, 462(2067), 725-748.

Zhang, F., Ye, B., Noda, T., Nakano, M., and Nakai, K. (2007). "Explanation of cyclic mobility of soils: Approach by stress-induced anisotropy." Soils Found., 47(4), 635-648.

Zhao, J. D., and Guo, N. (2013). "Unique critical state characteristics in granular media considering fabric anisotropy." Géotechnique, 63(8), 695-704.

Zhao, J. D., and Guo, N. (2015). "The interplay between anisotropy and strain localisation in granular soils: A multiscale insight." Géotechnique, in press. 